\title{
Phenomenology of top partners at the ILC
}

\section{Kyoungchul Kong and Seong Chan Park}

Theoretical Physics Department, Fermilab, Batavia, IL 60510, U.S.A., and

FPRD, School of Physics and Astronomy, Seoul National University,

Seoul 151-742, Korea

E-mail: kckong@fnal.gov, spark@mail.lns.cornell.edu, spark@phya.snu.ac.kn

ABSTRACT: The assumption of a new symmetry provides a nice explanation of the existence of dark matter and an elegant way to avoid the electroweak constraints. This symmetry often requires the pair production of new particles at colliders and it guarantees that cascade decays down to the lightest particle give rise to missing energy plus jets and leptons. For a long time, supersymmetry with the conserved R-parity was the only candidate for such signals. However, any new physics with this type of new symmetry may show up with similar signals and the discrimination between different models at the LHC is quite challenging. In this paper, we address the problem of discrimination between different models, more concretely, in the little Higgs theory with T-parity (LHT) and the supersymmetric theory with R-parity. We concentrate on the pair production of heavy top partners, e.g., T-odd quarks $\left(T_{-}\right)$in LHT and the scalar top quarks $(\tilde{t})$ in the MSSM at linear colliders (LC).

Keywords: Gauge Symmetry, Discrete and Finite Symmetries, Higgs Physics, Beyond Standard Model. 


\section{Contents}

1. Introduction 1

2. Set-up: Top quark sector of LHT 3

3. T-odd top pair production at LC 5

3.1 Production and decay

3.2 Backgrounds 9

3.3 Energy distribution of reconstructed top 10

3.4 Angular distribution of b-jet 11

4. Summary and conclusions 13

A. Left-right polarization asymmetry: $A_{L R}$

B. Forward-backward Asymmetry: $A_{F B}$

G. $T_{+}$production at the ILC

\section{Introduction}

Some of the most urgent theoretical issues in high energy physics, the natural realization of electroweak symmetry breaking (EWSB) and the study of its phenomenological implications at near future experiments have drawn big interests among particle physicists. Actually all the other sectors in the standard model of particle physics (SM) other than the Higgs sector have been experimentally well tested but the SM still has been regarded unnatural since the mass of the Higgs boson is quadratically sensitive to the cutoff scale $\left(\sim \Lambda_{\text {cut }}\right)$ which is supposed to be hierarchically larger than the weak scale $\left(\sim M_{W}\right)$.

Up to date, the low energy supersymmetry or its minimal realization (MSSM) accommodating the SM in its particle spectrum might have been regarded as the best motivated and successful example of a natural theory of EWSB. Even better, with the exact conservation of a discrete symmetry, dubbed R-parity, the lightest supersymmetric particle (LSP) in the MSSM can be a nice candidate of dark matter. The LSP (often the lightest neutralino $\left.\left(\tilde{\chi}_{0}^{1}\right)\right)$ appears in cascade decays of a super-particles but easily escapes the particle detectors thus the frequent appearance of missing energy signals can be thought as a genuine feature of the MSSM with R-parity. Very interestingly, this feature (the existence of a global symmetry and the stability of the lightest particle in the newly extended sector) is shared by recently suggested models beyond the SM. Among others, we are mainly interested in little Higgs theories [1, 2] (for reviews, see [3, 4.) with a parity, dubbed T-parity 
(LHT), by which the lightest new particle, a heavy photon $\left(A_{H}\right)$, is absolutely stable and any new particles in the extended sector beyond the SM can be produced only by a pair. Single production of the new particle is prohibited by the T-parity just like the R-parity in the MSSM.

In LHT, the Higgs field is a Pseudo-Goldstone boson. Higgs as a Goldstone boson has been considered since long time ago but its natural realization in the light of collective symmetry breaking was suggested quite recently. Once the Higgs mass is protected by several symmetries, as was the case in the little Higgs theories by construction, no oneloop quadratic mass could be induced. However the original formulation of little Higgs theories are severely constrained by electroweak precision data [5-7]. This mainly came from the fact that the newly introduced particles $(m \sim f)$ in the extended gauge and fermion sectors could directly mix with the standard model particles at the tree level without suitable symmetry protection. The most economic and elegant way out, up to date, is to introduce T-parity, in the theory and assign the most of new particles odd and the standard model particles even under the parity [8-10]. By doing so, no tree level mixing is allowed and electroweak constraints are greatly relieved [11]:

$$
f \gtrsim 500 \mathrm{GeV} \text {. }
$$

Once T-parity is introduced, the phenomenology of little Higgs models could be essentially similar to the low energy supersymmetry with R-parity [12]. The lightest T-odd particle cannot further decay to the single standard model particle similarly to the lightest neutralino $\tilde{\chi}_{0}^{1}$ in supersymmetric models. Because of this similarity, little Higgs models can "fake" the supersymmetric signals at the near future colliders, such as the CERN Large Hadron Collider (LHC). Future linear colliders, such as ILC and TESLA, could help to provide valuable distinctions between MSSM and the other models, e.g., LHT. That's the main motivation of this study.

More specifically, we would concentrate on the phenomenology of T-odd, new top quark (T) which is the lightest among all the newly introduced fermions, in most realization of little Higgs models with T-parity. The decay signal of T-quark $\left(\mathrm{T} \rightarrow t A_{H}\right)$ can fake the signal of the scalar top quark $\left(\tilde{t} \rightarrow t \tilde{\chi}_{0}^{1}\right)$. Note that provided the mass of the scalar top is heavy enough $\left(m_{\tilde{t}}>m_{t}+m_{\tilde{\chi}_{0}^{1}}\right)$, scalar top can dominantly decay to the standard model top quark and the neutralino.

This paper is organized as follows. In section 2, we setup the model of little Higgs with T-parity. The mass and the gauge couplings of T-quark are specified and the relevant Feynman rules are derived. In section 3 , we study the T-quark pair production by electronpositron collision $\left(e^{-} e^{+} \rightarrow T \bar{T}\right)$. We first calculate the total cross section of T-quark production and compare it with the one of the scalar top pair production when their masses are set to be the same. The angular distributions for produced leptons taking the cascade decay of top quark is found to give a clear distinction between the cases with Tquark (fermion) in LHT and scalar top quark in the MSSM. The use of the polarization of initial electron and positron is also discussed. Summary will be given in the last section. 


\section{Set-up: Top quark sector of LHT}

In this section, we will set up the top quark sector of little Higgs model with T-parity. We restrict ourselves to the $\mathrm{SU}(5) / \mathrm{SO}(5)$ realization of little Higgs mechanism to be specific [2]. There have been lots of phenomenological studies of the littlest Higgs models without [13]16] and with T-parity [17-27.

To cancel out the 1-loop contribution of the standard model top quark, the Yukawa sector of the third generation need to be extended. To incorporate the collective symmetry breaking pattern, the third generation quarks should be elevated to the complete $\mathrm{SU}(3)$ representations:

$$
Q_{1}=\left(\begin{array}{c}
q_{1} \\
T_{1} \\
0
\end{array}\right), \quad Q_{2}=\left(\begin{array}{c}
0 \\
T_{2} \\
q_{2}
\end{array}\right)
$$

where $q_{1} \sim \mathbf{2}_{1 / 30} \times \mathbf{1}_{2 / 15}, q_{2} \sim \mathbf{1}_{2 / 15} \times \mathbf{2}_{1 / 30}$ are doubles under $[\mathrm{SU}(2) \times \mathrm{U}(1)]_{1} \times[\mathrm{SU}(2) \times$ $\mathrm{U}(1)]_{2}$ gauge symmetry of $\mathrm{SU}(5)$ subgroup and $T_{1} \sim \mathbf{1}_{8 / 15} \times \mathbf{1}_{2 / 15}$ and $T_{2} \sim \mathbf{1}_{2 / 15} \times \mathbf{1}_{8 / 15}$ are singlets. Under T-parity operation, $Q_{1} \rightarrow-\Sigma_{0} Q_{2}$ where $\Sigma_{0}$ is the vev of an $\mathrm{SU}(5)$ symmetric tensor by which $\mathrm{SU}(5)$ global symmetry is broken down to $\mathrm{SO}(5)$ at the energy scale $\sim f$. On top of the standard model top quark, there are new T-even and T-odd quarks:

$$
T_{ \pm}=\frac{1}{\sqrt{2}}\left(T_{1} \mp T_{2}\right)
$$

and their masses are

$$
M_{T_{+}}=\sqrt{\lambda_{1}^{2}+\lambda_{2}^{2}} f, \quad M_{T_{-}}=\lambda_{2} f,
$$

where $\lambda_{i} \simeq 1$ parameters are introduced to give the Yukawa couplings. We can immediately notice that T-odd quark is always lighter than T-even quark and its production would be important at the near future colliders. The mass of standard model top quark is given by

$$
M_{t}=\frac{\lambda_{1} \lambda_{2} v}{\sqrt{\lambda_{1}^{2}+\lambda_{2}^{2}}}
$$

where $v \simeq 246 \mathrm{GeV}$ is the measured vacuum expectation value of the Higgs. The gauge interaction with $Z$ boson and photon $\gamma$ are immediately read out. Because T's are SU(2) singlets, there is no coupling with $W$ boson.

$$
\mathcal{L}=\bar{T}_{-}\left[\frac{2}{3} \gamma_{\mu}\left(e A^{\mu}-\frac{g}{c_{w}} s_{w}^{2} Z^{\mu}\right)\right] T_{-} .
$$

T-odd quark $\left(T_{-}\right)$could decay to the standard model top quark and newly introduced neutral gauge bosons, $Z_{H}$ or $A_{H}$ through

$$
\mathcal{L}=\bar{T}_{-}\left[-\frac{2 g^{\prime} c_{\lambda}}{5} \gamma_{\mu}\left(\left(P_{R}+c_{\lambda} \frac{v}{f} P_{L}\right) A_{H}^{\mu}+x_{h} \frac{v^{2}}{f^{2}} P_{R} Z_{H}^{\mu}\right)\right] t
$$



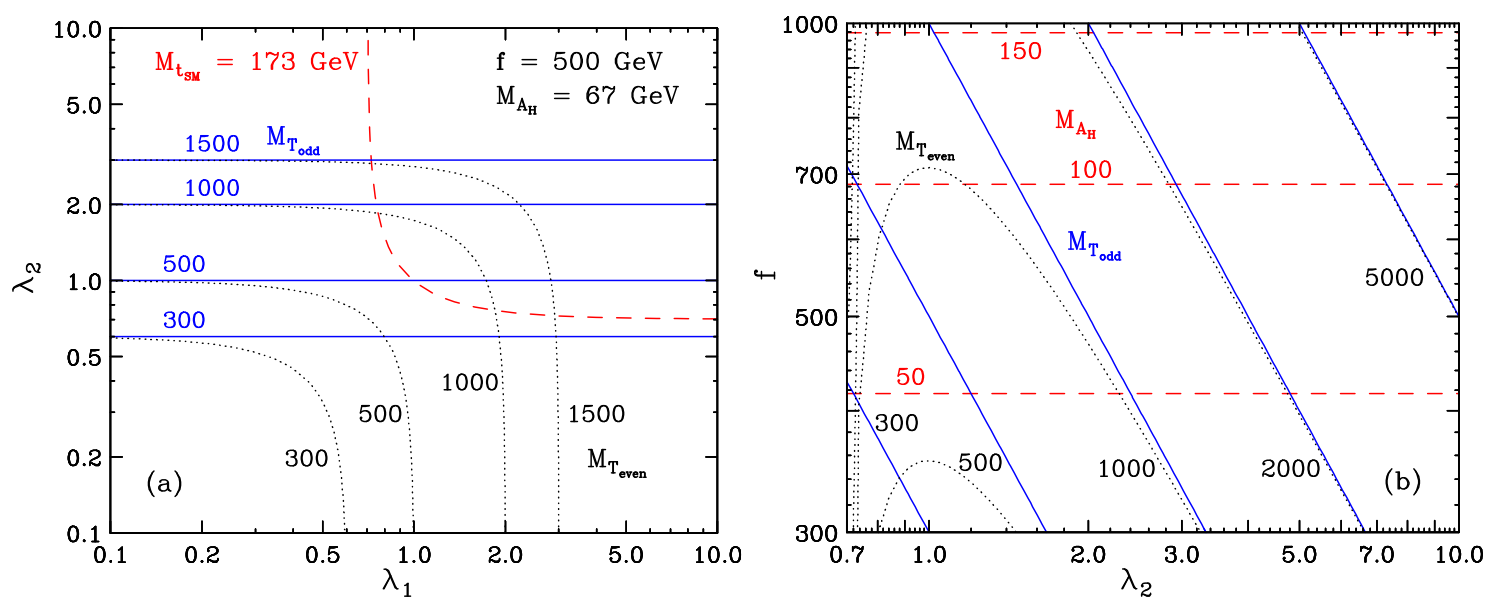

Figure 1: The masses of T-even quark (black, dotted) and T-odd quark (blue, solid) for given (a) $f=500 \mathrm{GeV}$ and (b) $M_{t_{\mathrm{SM}}}=173 \mathrm{GeV}$. In (a), a red dashed line shows a contour with $M_{t_{\mathrm{SM}}}=173$ in the plane of $\lambda_{2}$ versus $\lambda_{1} 1$. In (b), $\lambda_{1}$ is determined assuming $M_{t_{\mathrm{SM}}}=173 \mathrm{GeV}$.

where the mixing parameters $c_{\lambda}$ and $x_{h}$ are defined as [12]:

$$
\begin{aligned}
c_{\lambda} & \equiv \frac{\lambda_{1}}{\sqrt{\lambda_{1}^{2}+\lambda_{2}^{2}}}, \\
x_{h} & \equiv \frac{5 g g^{\prime}}{4\left(5 g^{2}-g^{\prime 2}\right)} .
\end{aligned}
$$

Since $Z_{H}$ acquires heavy mass $\left(M_{Z_{H}}^{2}=g^{2}\left(f^{2}-v^{2} / 4\right)\right)$ but the other one $A_{H}$ does not, $\left(M_{A_{H}}^{2}=g^{\prime 2}\left(f^{2} / 5-v^{2} / 4\right) \simeq 0.16^{2} f^{2}\right), T_{-} \rightarrow t A_{H}$ dominates the decay or $B R\left(T_{-} \rightarrow\right.$ $\left.t A_{H}\right) \simeq 1$ is a good approximation.

We show the masses of T-odd and T-even particles in figure 1. We fix $f=500 \mathrm{GeV}$ and $M_{t_{\mathrm{SM}}}=173 \mathrm{GeV}$ in figure 1(a). Then the mass of the lightest T-odd particle (LTP) is $M_{A_{H}}=67 \mathrm{GeV}$. A red dashed contour line shows SM top mass in the plane of $\lambda_{2}$ versus $\lambda_{1}$ and blue solid lines represent masses of T-odd top partner for $M_{T_{\text {odd }}}=300,500,1000,1500 \mathrm{GeV}$. Black dotted lines represent the masses for the T-even partners for $M_{T_{\text {even }}}=300,500,1000,1500 \mathrm{GeV}$. However $\lambda_{1}$ is not an independent parameter if we assume top quark mass. From eq. 2.4, it is determined by $\lambda_{2}$ and $M_{T}$ as follows,

$$
\begin{aligned}
\lambda_{1} & =\frac{\lambda_{2}}{\sqrt{\lambda_{2}^{2} \frac{v^{2}}{M_{t}^{2}}-1}}, \\
\lambda_{2} & >\frac{M_{t}}{v} \sim 0.7, \\
f & >\sqrt{\frac{5}{4}} v \sim 275 \mathrm{GeV} .
\end{aligned}
$$

Figure 1(b) shows $M_{T_{\text {odd }}}$ in the plane of f versus $\lambda_{2}$ assuming $\lambda_{1}$ is determined. $0.7<\lambda_{2}<1$ and $400 \mathrm{GeV}<f<700 \mathrm{GeV}$ where $M_{T_{\text {odd }}}<500 \mathrm{GeV}$ is an interesting region for the pair production of T-odd partner at the ILC with $\sqrt{s} \leq 1 \mathrm{TeV}$. Note that precision electroweak 

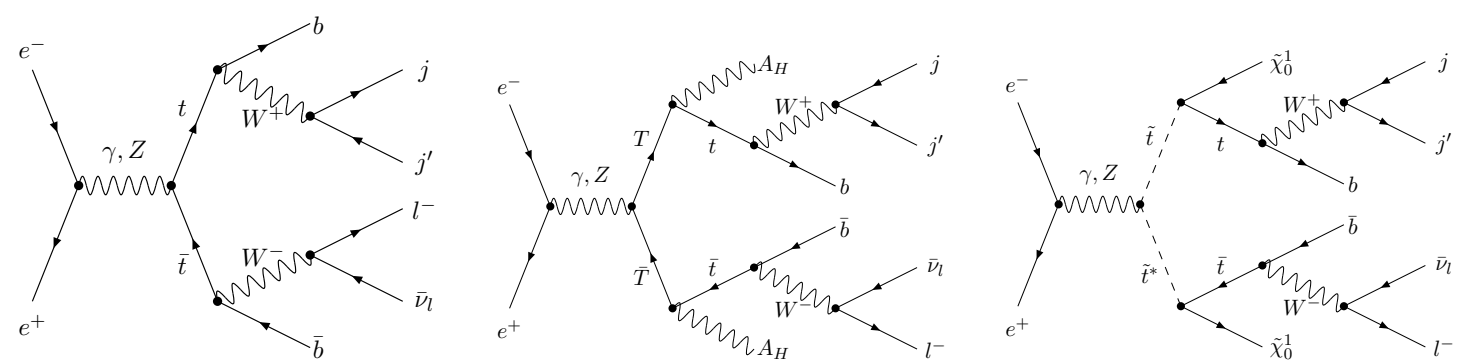

Figure 2: Feynman diagrams for pair productions of top quark in the SM, T-odd quarks/scalar top quarks and their subsequent decay to $t A_{H} / t \tilde{\chi}_{1}^{0}$ in LHT and MSSM, respectively. The final state signals are identical $\left(l^{-} j j b b+\mathbb{E}_{T}\right)$.

constraints on this model consistently allows values for $f$ as low as $500 \mathrm{GeV}$ without fine tuning [12].

We would add some comments about possible signals of T-even top partner at the ILC and LHC. At the LHC, a single production of the T-even top partner and a quark-jet is possible through T-channel exchange diagram of W-boson [14, 15]. Unfortunately the same production mechanism does not work at the ILC because the T-even top partner does not directly couple with electron and W-boson. There remains a possibility that T-even top partners to be produced by pair through $s$-channel photon and Z-boson exchange diagrams. But it is highly suppressed or disallowed by kinematics in most of parameter space we are considering. For details, see the appendix C. Considering all these issues, we would like to concentrate on phenomenology of T-parity odd partners $\left(T_{-}\right)$. It is also fair to assume that the top partner is odd under a discrete symmetry since we plan to compare with stop in SUSY. We will use a study point, $\lambda_{2}=0.8$ and $f=500 \mathrm{GeV}$, for which pair production of Teven partners is not allowed at ILC with $\sqrt{s}=1 \mathrm{TeV}$. Our analysis can be also applied to fermionic partner of top in any models with a discrete symmetry such as KK-top in UED model.

\section{T-odd top pair production at LC}

\subsection{Production and decay}

The top quark pair production mechanisms (with or without the missing energy) in the SM, LHT and MSSM are depicted in figure 2. The pair production cross section of T-odd fermion is calculated based on the interaction given in section 2 .

$$
\begin{aligned}
\frac{d \sigma}{d \cos \theta} & =\frac{N_{c} \beta s}{128 \pi}\left[\mathcal{A}(1+\beta \cos \theta)^{2}+\mathcal{B}(1-\beta \cos \theta)^{2}+\mathcal{C}\left(1-\beta^{2}\right)\right], \\
\sigma & =\frac{N_{c} \beta s}{64 \pi}\left[(\mathcal{A}+\mathcal{B})\left(1+\frac{1}{3} \beta^{2}\right)+\mathcal{C}\left(1-\beta^{2}\right)\right],
\end{aligned}
$$

where $N_{c}=3$ is the number of color charges, $\beta=\sqrt{1-\frac{4 m^{2}}{s}}$ is the velocity of the final state fermion and the effective product of couplings and propagator factors are conveniently 
defined as

$$
\mathcal{A}=\left(\left|G_{L L}\right|^{2}+\left|G_{R R}\right|^{2}\right), \mathcal{B}=\left(\left|G_{L R}\right|^{2}+\left|G_{R L}\right|^{2}\right), \mathcal{C}=2 R e\left(G_{L L} G_{L R}^{*}+G_{R R} G_{R L}^{*}\right)
$$

where $G_{A B}$ ( $A$ and $B$ denote Left and Right-handed chiralities.) are given as

$$
G_{A B}(s)=\sum_{X} \frac{g_{A}(X \rightarrow \ell \bar{\ell}) g_{B}(X \rightarrow f \bar{f})}{s-M_{X}^{2}+i M_{X} \Gamma_{X}},
$$

summed over all contributing gauge bosons, in this case $X=\gamma, Z$ (see [28] for detail.). $\gamma$ contribution is dominant and $Z$ contribution is roughly $10 \%$ of the total cross section. The cross term is negligible (smaller by two order of magnitude). The cross section is calculated with CalcHEP [29] and cross-checked with MadGraph/MadEvent [30, 31]. One important point we should notice here is that because T-quark is a vector-like fermion, $G_{L L}=G_{L R}$ and $G_{R R}=G_{R L}$ :

$$
\begin{aligned}
& G_{L L}=G_{L R}=\frac{(-e)\left(\frac{2}{3} e\right)}{s}+\frac{g_{L} g_{Z T \bar{T}}}{s-M_{Z}^{2}} \\
& G_{R R}=G_{R L}=\frac{(-e)\left(\frac{2}{3} e\right)}{s}+\frac{g_{R} g_{Z T \bar{T}}}{s-M_{Z}^{2}} .
\end{aligned}
$$

Here $g_{L}$ and $g_{R}$ are the couplings between $Z$ and $e^{+} e^{-}$given as $g_{L / R}=\frac{e}{s_{W} c_{W}}\left(I_{3}-Q_{e} s_{W}^{2}\right)$ where $I_{3}=-\frac{1}{2}$ is the third component of weak iso-spin of the left-chiral electron, $Q_{e}=$ -1 its charge and the coupling between $Z$ and $T \bar{T}$ is given as $g_{Z T \bar{T}}=-\frac{2}{3} \frac{g}{c_{W}} s_{W}^{2}$. As a consequence, we get a simple relation between $\mathcal{A}, \mathcal{B}$ and $\mathcal{C}$ :

$$
\mathcal{A}=\mathcal{B}=\frac{1}{2} \mathcal{C} \text {. }
$$

What can we learn from this observation? First of all, as is explicitly shown in the appendix, the forward-backward asymmetry vanishes. This is actually a generic feature of a vectorlike fermion. Based on the precise measurement on $A_{F B}$ asymmetry, one will be able to clearly prove that the produced T-quark is a vector-like fermion even though its direct measurement might be challenging. Furthermore, the Left-Right asymmetry, $A_{L R}$ can provide a useful information about the couplings between T-quark and vector bosons $\gamma$ and $Z$ (see appendix).

In figure $3(a)$, we show the pair production cross section of T-odd partner as a function of $f$ at a $1 \mathrm{TeV}$ linear collider for various values of $\lambda_{2}$. As we mentioned earlier, photon dominantly contributes to total cross section. However, $Z$ contribution also becomes important when polarized beams are used and figure $3(\mathrm{~b})$ shows production cross section with polarized beams for $f=500 \mathrm{GeV}$ and $\lambda_{2}=0.8\left(M_{T_{\text {odd }}}=400 \mathrm{GeV}\right)$. Polarized beams may be used to confirm the nature of T-odd partner by measuring cross sections with different polarizations of the beams. As $\lambda_{2}$ increases, the corresponding cross section decreases since the mass of T-odd partner is proportional to $\lambda_{2}$. The pair production of T-odd partner with $f \geq 700 \mathrm{GeV}$ at such a collider is not allowed as shown in figure 1(b). Solid lines represent cross section without the initial state radiation (ISR) while the dotted lines are ISR corrected. Generally the cross section at the linear collider is given by 

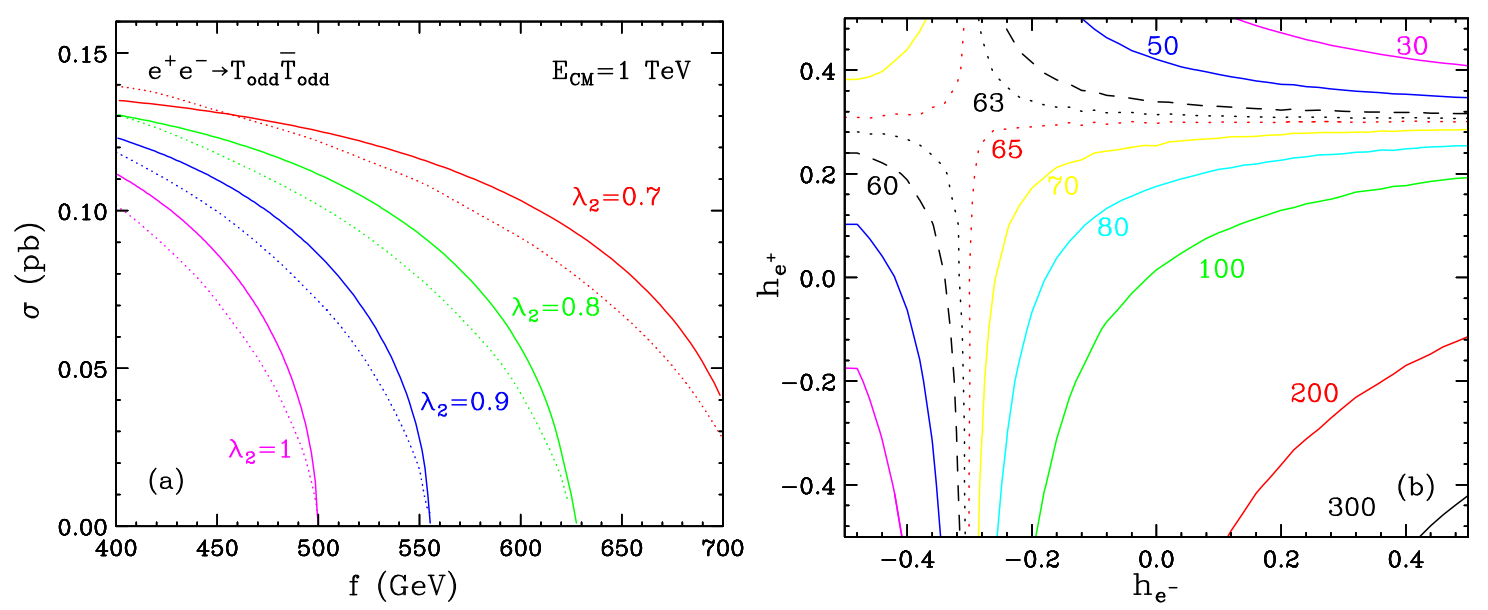

Figure 3: Cross sections for T-odd quark pair production (a) with unpolarized beam for various values of $\lambda_{2}$ in $\mathrm{pb}$ and (b) with the initial helicity states of electron and positron in fb. The dotted lines in (a) are ISR-corrected.

$$
\begin{aligned}
\sigma\left(e^{+} e^{-} \rightarrow T \bar{T}\right)= & \int d x_{1} d x_{2}\left[f_{e / e}\left(x_{1}\right) f_{e / e}\left(x_{2}\right) \hat{\sigma}\left(e^{+} e^{-} \rightarrow T \bar{T}\right)\right. \\
& \left.+\left(f_{\gamma / e}^{\text {brem }}\left(x_{1}\right)+f_{\gamma / e}^{\text {beam }}\left(x_{1}\right)\right)\left(f_{\gamma / e}^{\text {brem }}\left(x_{2}\right)+f_{\gamma / e}^{\text {beam }}\left(x_{2}\right)\right) \hat{\sigma}(\gamma \gamma \rightarrow T \bar{T})\right] \\
& \approx \int d x_{1} d x_{2}\left[f_{e / e}^{\text {brem }}\left(x_{1}\right) f_{e / e}^{\text {brem }}\left(x_{2}\right) \hat{\sigma}\left(e^{+} e^{-} \rightarrow T \bar{T}\right)\right] .
\end{aligned}
$$

Here $f_{\gamma / e}(x)$ is the parton distribution function for finding a photon inside the electron beam and $f_{e / e}(x)$ is the parton distribution function for finding an electron inside the electron beam. $f_{e / e}(x)$ is given by the convolution of bremstrahlung (or ISR) and beamstrahlung,

$$
f_{e / e}(x)=\int_{x}^{1} \frac{d z}{z} f_{e}^{\text {brem }}\left(\frac{x}{z}\right) f_{e}^{\text {beam }}(z)
$$

where $f_{e}^{\text {beam }}(x)$ is the beamstrahlung distribution function of the electron and $f_{e}^{\text {brem }}$ is the bremstrahlung distribution function of the electron. CalcHEP [29] realizes the breamstrahlung function with the expression in [32, 33],

$$
f_{e}^{\operatorname{brem}}(x)=e^{\beta(3 / 4-\gamma)} \beta(1-x)^{\beta-1} \frac{\left(1+x^{2}\right)-\beta\left(\left(1+3 x^{2}\right) \log (x) / 2+(1-x)^{2}\right) / 2}{2 \Gamma(1+\beta)},
$$

where $\beta=\frac{\alpha}{\pi}\left(2 \log \left(\frac{Q}{m_{e}}\right)-1\right), \gamma=0.5772156649$ is the Euler constant, $\Gamma$ is the gamma function, $m_{e}$ is the electron mass, and $\alpha=1 / 137.0359895$ is the fine structure constant. We take ISR scale, $Q=\sqrt{s}$. We find that in our study the beamstrahlung effect (for the small beamstrahlung parameter) in the cross sections and the contribution from $\sigma(\gamma \gamma \rightarrow T \bar{T})$ are small enough to ignore (See [34, 35] for the beamstrahlung effect at the high energy linear colliders such as CLIC). In figure 3(a), we can notice that the inclusion of ISR gives smaller cross sections (except for $\lambda_{2}=0.7$ ) since the beam loses its energy. However for the case of the light T-odd partner (i.e., $\lambda_{2} \sim 0.7$ and $f<500 \mathrm{GeV}$ ), the ISR increases cross sections 


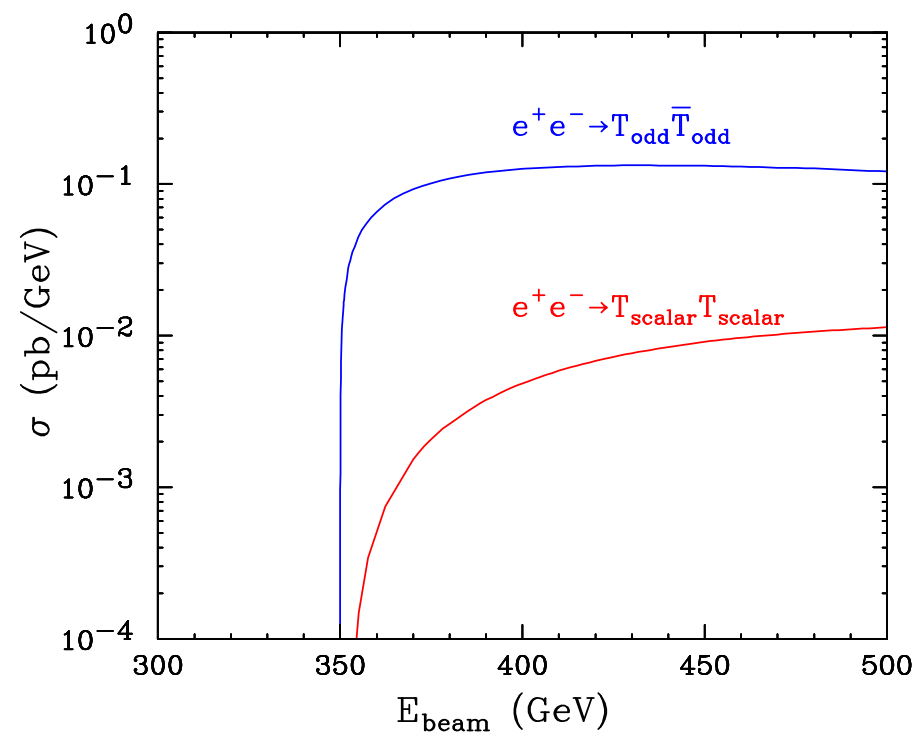

Figure 4: Pair production cross section in LHT (blue) and SUSY (red) near the threshold

since the energy loss of the beam results in getting closer to the pair production threshold which behaves as $\sigma_{L H} \sim \beta$ where $\beta=\sqrt{1-\frac{4 M_{T_{\text {odd }}}^{2}}{s}}$. In the case of pair production of scalar partners (i.e., stop), the threshold rises as $\sigma_{\mathrm{SUSY}} \sim \beta^{3}$. The production of fermion (T-odd partner) and the production of scalar (stop) have different behavior near the threshold as shown in figure 4 . It is well known that in the massless limit of new particles, the cross section ratio converges to a factor of 4 due to the fact that there are two different helicity states in LH while in SUSY, stop is a scalar particle [36-38]. Fermions prefer the forward or backward direction when they are produced at $e^{+} e^{-}$collider,

$$
\left(\frac{d \sigma}{d \cos \theta}\right)_{L H} \sim 2\left(1+\cos ^{2} \theta\right),
$$

while scalar particles are produced more in the central region,

$$
\left(\frac{d \sigma}{d \cos \theta}\right)_{\mathrm{SUSY}} \sim\left(1-\cos ^{2} \theta\right) .
$$

These particles go through further decays and we consider semi-leptonic decay of SM top. We assume that we know the mass and momentum of $W$ and $t$ from two jets and one b-jet. Figure 5 shows angular distributions in LH and MSSM in the semi-leptonic channel. The distribution for MSSM is similar to the angular distribution of the scalar production. We see clear difference between two distributions. When top partners are produced, Todd fermions prefer forward or backward direction while stops are mainly produced in the central region, as explained above. There is no forward-backward asymmetry at this production level. Now they continue to decay into to SM top to $W_{\mu}$ and $b$. As noticed in [41], in the case of SM top pair production at LC, the forward-backward asymmetry appears due to huge interference between $\gamma$ and $Z$ (negative in backward and positive in 

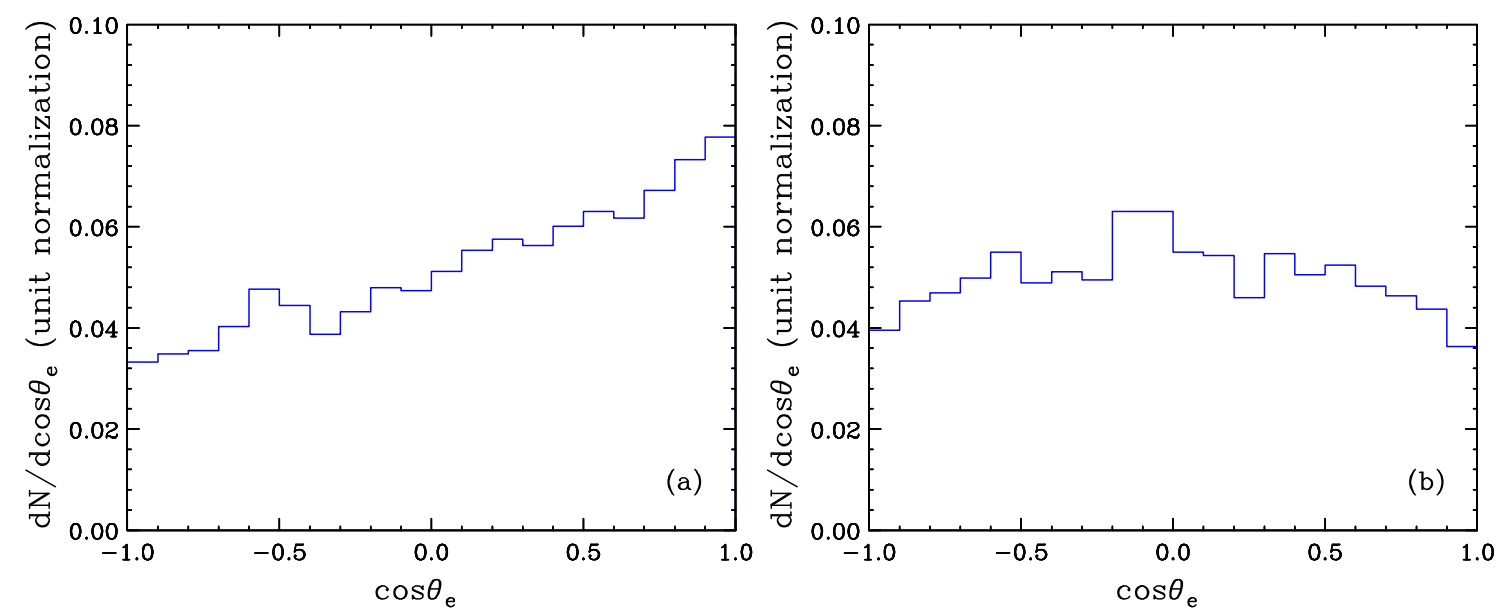

Figure 5: The angular distribution of final state lepton $\left(\ell^{-}\right)$for LHT and MSSM, respectively. In LHT, leptons follow the angular distribution of the parent T-odd quarks and highly anisotropic. However in MSSM, mainly because of the characteristics of scalar particle production, the angular distribution is roughly $\left(1-\cos ^{2} \theta\right)$. (PYTHIA 40 also gives similar distribution in the case of MSSM.)

forward region), although there is no FB asymmetry at all in $\gamma$-mediation and small FB asymmetry appears in the $Z$-mediation. This characteristic feature is carried in the decay of T-odd and the angular distribution of top at LC in the production just looks like angular distribution of the lepton in the case of LH. However, in the case of SUSY, the angular distribution is not spoiled as much as LH case since they are produced in the central region. (See 42, 25, 43-46] for the discrimination between SUSY and other new physics such as $\mathrm{UED} / \mathrm{LH}$ at the LHC in a particular cascade decay. These studies seem to either require large luminosity or depend on certain assumptions.)

\subsection{Backgrounds}

We list possible backgrounds for our signal in table 1. The dominant backgrounds are $t \bar{t}$ and $\nu_{e} e^{+} W^{-} Z$. $t \bar{t}$ can be easily distinguished from our signal in the energy distribution of SM top since $E_{\text {top }}=\frac{\sqrt{s}}{2}$ in SM while LHT or SUSY shows a flat distribution over wide range of $E_{\text {top }}$ as shown in figure 6. In fact, the upper edge $\left(E_{+}\right)$is always less than $E_{\text {top }}=\frac{\sqrt{s}}{2}$ and signal is well separated from this background. A background $t \bar{t} Z$ is included when $t \bar{t} \nu \bar{\nu}$ is estminated. The last three backgrounds have two b-jets from $Z$ decay and therefore they can be eliminated by imposing relevant cut on invariant mass of two b-jets. The rest of background are much smaller than our signal cross section, $\sigma\left(e^{+} e^{-} \rightarrow T \bar{T} \rightarrow 2 j+2 b+\ell+\mathbb{E}_{T}\right)=29 \mathrm{fb}$ assuming $\mathcal{O}\left(\sigma\left(e^{+} e^{-} \rightarrow t \bar{t}\right)\right) \sim 100 \mathrm{fb}$. These backgrounds are also ignored in supersymmetric case (see 477 for details) with similar reasons. However our case is even better since the pair production cross section of two fermions (T-odd) is much larger than the pair production cross section of two scalars (stop), as discussed in the previous section. With the signal cross section and the expected ILC luminosity $\mathcal{L}=500$ or $1000 \mathrm{fb}^{-1}$, we can easily get large number events even if we 


$$
\begin{array}{|l|l|}
\hline \sigma\left(e^{+} e^{-} \rightarrow t \bar{t}\right)=173 & \sigma\left(e^{+} e^{-} \rightarrow t \bar{t} \rightarrow 2 j+2 b+\ell+\mathbb{E}_{T}\right)=50 \\
\sigma\left(e^{+} e^{-} \rightarrow t \bar{t} \nu_{e} \bar{\nu}_{e}\right)=0.74 & \sigma\left(e^{+} e^{-} \rightarrow t \bar{t} \nu_{e} \bar{\nu}_{e} \rightarrow 2 j+2 b+\ell+\mathbb{E}_{T}\right)=0.22 \\
\sigma\left(e^{+} e^{-} \rightarrow t \bar{t} \nu_{\mu} \bar{\nu}_{\mu}\right)=0.317 & \sigma\left(e^{+} e^{-} \rightarrow t \bar{t} \nu_{\mu} \bar{\nu}_{\mu} \rightarrow 2 j+2 b+\ell+\mathbb{E}_{T}\right)=0.09 \\
\sigma\left(e^{+} e^{-} \rightarrow Z W^{+} W^{-}\right)=56.8 & \sigma\left(e^{+} e^{-} \rightarrow Z W^{+} W^{-} \rightarrow 2 j+2 b+\ell+\mathbb{E}_{T}\right)=2.5 \\
\sigma\left(e^{+} e^{-} \rightarrow \nu_{e} e^{+} W^{-} Z\right)=165.45 & \sigma\left(e^{+} e^{-} \rightarrow \nu_{e} e^{+} W^{-} Z \rightarrow 2 j+2 b+\ell+\mathbb{E}_{T}\right)=2.68 \\
\sigma\left(e^{+} e^{-} \rightarrow \nu_{\mu} \mu^{+} W^{-} Z\right)=6.3 & \sigma\left(e^{+} e^{-} \rightarrow \nu_{\mu} \mu^{+} W^{-} Z \rightarrow 2 j+2 b+\ell+\mathbb{E}_{T}\right)=0.65 \\
\hline
\end{array}
$$

Table 1: SM backgrounds (in fb) in semi-leptonic channel are estimated using MadEvent/MadGraph and cross-checked with CalcHEP. We used $B R(Z \rightarrow \nu \bar{\nu})=0.6666, B R\left(t \rightarrow W^{+} b\right)=1, B R\left(W^{ \pm} \rightarrow\right.$ $\left.j j^{\prime}\right)=0.6796, B R\left(W^{ \pm} \rightarrow \ell^{ \pm} \nu_{\ell}\right)=0.1068$ and $B R(Z \rightarrow b \bar{b})=0.1514$.

lose some of events by imposing cuts to remove SM backgrounds. Therefore we can safely ignore backgrounds in our study.

\subsection{Energy distribution of reconstructed top}

In the hadronic (semi-leptonic channel), we can reconstruct momenta of two (one) top quarks and look at the energy distribution of top. There are two endpoints given by

$$
E_{+/-}=\frac{\gamma}{2 M_{T}}\left(M_{T}^{2}-M_{N}^{2}+m_{t}^{2} \pm \beta \sqrt{\left[M_{T}^{2}-\left(M_{N}+m_{t}\right)^{2}\right]\left[M_{T}^{2} !-\left(M_{N}-m_{t}\right)^{2}\right]}\right),
$$

where $\beta=\sqrt{1-\frac{4 M_{T}^{2}}{s}}, \gamma=\frac{1}{\sqrt{1-\beta^{2}}}, M_{T}$ denotes the mass for T-odd fermion or stop, $M_{N}$ for $A_{H}$ or $\tilde{\chi}_{1}^{0}$ and $m_{t}$ for SM top. From the measurement of these two end points, we get two unknown masses

$$
\begin{aligned}
& M_{T}=\sqrt{s} \frac{\sqrt{E_{+} E_{-}}}{E_{-}+E_{+}} \frac{1}{\sqrt{2}} \sqrt{1+\frac{m_{t}^{2}}{E_{+} E_{-}}}+\sqrt{\left(1-\frac{m_{t}^{2}}{E_{+}^{2}}\right)\left(1-\frac{m_{t}^{2}}{E_{-}^{2}}\right)}, \\
& M_{N}=M_{T} \sqrt{1-\frac{2\left(E_{+}+E_{-}\right)}{\sqrt{s}}+\frac{m_{t}^{2}}{M_{T}^{2}}} .
\end{aligned}
$$

This method is usually discussed in the slepton pair production and taking top quark mass to be zero, we recover well known formulas

$$
\begin{aligned}
E_{+/-} & =\frac{\sqrt{s}}{4}\left(\frac{M_{T}^{2}-M_{N}^{2}}{m_{T}^{2}}\right)\left(1 \pm \sqrt{1-\frac{4 M_{N}^{2}}{s}}\right) \\
M_{T} & =\sqrt{s} \frac{\sqrt{E_{+} E_{-}}}{E_{-}+E_{+}} \\
M_{N} & =M_{T} \sqrt{1-2 \frac{E_{+}+E_{-}}{\sqrt{s}}} .
\end{aligned}
$$

The accuracy of this method depends on how well we can reconstruct top quark momentum. For our study point, $m_{T}=400 \mathrm{GeV}$ and $m_{A_{H}}=66.76 \mathrm{GeV}$, we get two endpoints at $E_{-}=174 \mathrm{GeV}$ and $E_{+}=406 \mathrm{GeV}$, as shown in figure 6. The distribution gets smeard near $E_{+}$due to ISR at LC. 


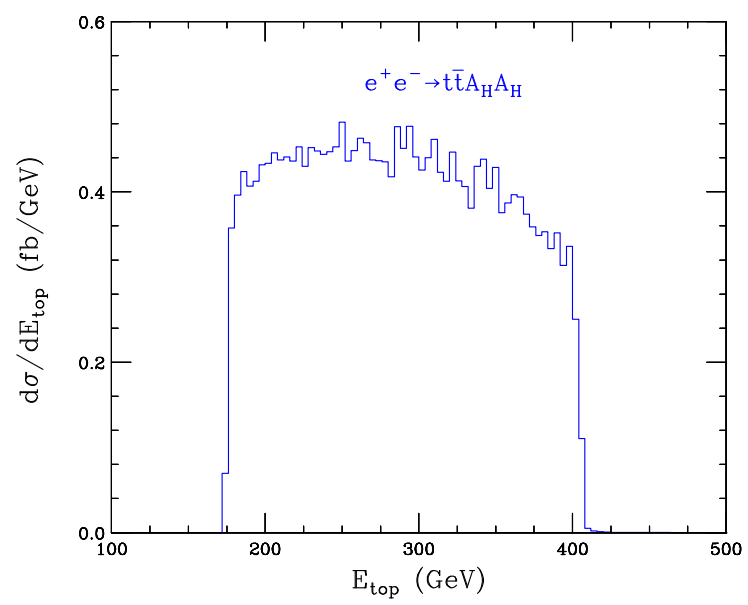

Figure 6: The energy distribution of top quark

\subsection{Angular distribution of b-jet}

In [47], it was noticed that the information about the stop and the LSP is imprinted in the helicity of the top quark in the stop decay, and hence the measurement of the helicity of the top quark provides some knowledge of these particles. We follow the same analysis given in [47]. The Lagrangian is given by

$$
\mathcal{L}=\bar{T}_{-} \gamma_{\mu}\left(f_{L} P_{L}+f_{R} P_{R}\right) A_{H}^{\mu} t+H . C .,
$$

where $P_{L / R}=\frac{1 \mp \gamma_{5}}{2}$. Using the spin vector method, the spin vector $S^{\mu}$ for the top quark in the decay process, $T \rightarrow t A_{H}$ is given by

$$
\begin{aligned}
\frac{1}{2} N\left(\not P_{t}+m_{t}\right)\left(1+\$ \gamma_{5}\right) \equiv & \left(\not P_{t}+m_{t}\right) \gamma^{\mu}\left(f_{L}^{*} P_{L}+f_{R}^{*} P_{R}\right)\left(\not P_{T}+M_{T}\right) \gamma^{\nu}\left(f_{L} P_{L}+f_{R} P_{R}\right)\left(\not P_{t}+m_{t}\right) \\
& \times\left(-g_{\mu \nu}+\frac{P_{A_{H}}^{\mu} P_{A_{H}}^{\nu}}{M_{A_{H}}^{2}}\right),
\end{aligned}
$$

with $P_{t}, P_{A_{H}}$ and $P_{T}$ being four-momenta of the top quark, the LTP and the T-odd top, respectively. From this equation, $N$ and $S^{\mu}$ are obtained as follows.

$$
\begin{aligned}
N= & \left(\left|f_{L}\right|^{2}+\left|f_{R}\right|^{2}\right)\left[\left(M_{T}^{2}+m_{t}^{2}-M_{A_{H}}^{2}\right)+\frac{\left(M_{T}^{2}-m_{t}^{2}+M_{A_{H}}^{2}\right)\left(M_{T}^{2}-m_{t}^{2}-M_{A_{H}}^{2}\right)}{M_{A_{H}}^{2}}\right] \\
& -12 m_{t} M_{T} \operatorname{Re}\left(f_{L}^{*} f_{R}\right), \\
N S^{\mu}= & \frac{\left|f_{L}\right|^{2}-\left|f_{R}\right|^{2}}{m_{t} M_{A_{H}}^{2}}\left[\left(\left(M_{T}^{2}-m_{t}^{2}\right)^{2}+3 M_{A_{H}}^{2}\left(M_{T}^{2}-m_{t}^{2}\right)-2 M_{A_{H}}^{4}\right) P_{t}^{\mu}\right. \\
& \left.\quad-2 m_{t}^{2}\left(M_{T}^{2}-m_{t}^{2}+2 M_{A_{H}}^{2}\right) P_{A_{H}}^{\mu}\right] .
\end{aligned}
$$

For the process $T \rightarrow t A_{H} \rightarrow W^{+} b A_{H}$, the angular distribution of the b-jet in the rest frame of the top quark is given by

$$
\frac{1}{\Gamma_{T}} \frac{d \Gamma_{T}}{d \cos \theta_{\mathrm{tb}}} \equiv \frac{1}{2}\left(1+A_{b} \cos \theta_{\mathrm{tb}}\right),
$$



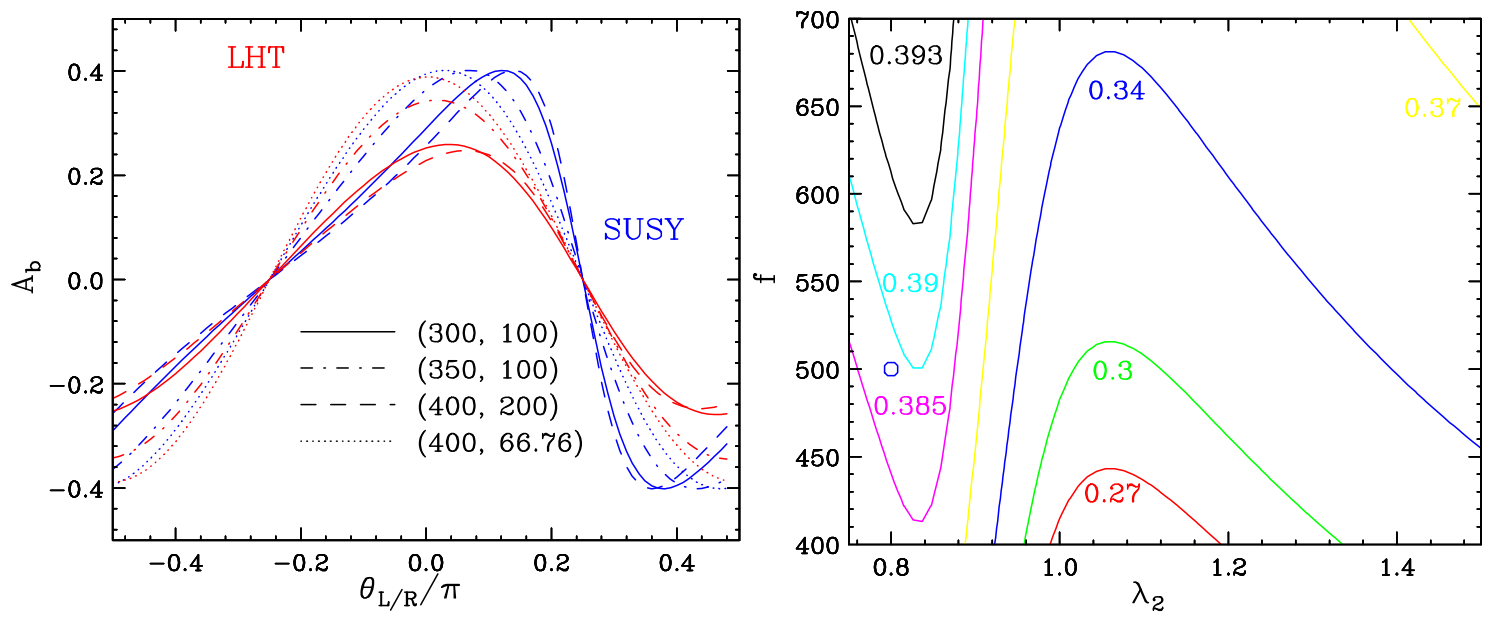

Figure 7: (a) $A_{b}$ as a function of $\tan \theta_{L / R}=f_{L} / f_{R}$ in SUSY (blue) and LHT (red). The masses of top-partner $(\tilde{t}$ or $T)$ and $\operatorname{LSP}\left(\tilde{\chi}_{1}^{0}\right) / \operatorname{LTP}\left(A_{H}\right)$ are taken to be $M_{T} / M_{\tilde{t}}=300 \mathrm{GeV}$ and $M_{\tilde{\chi}_{1}^{0}} / M_{A_{H}}=$ $100 \mathrm{GeV}$ (solid line), $M_{T} / M_{\tilde{t}}=350 \mathrm{GeV}$ and $M_{\tilde{\chi}_{1}^{0}} / M_{A_{H}}=100 \mathrm{GeV}$ (dash-dotted line), $M_{T} / M_{\tilde{t}}=$ $400 \mathrm{GeV}$ and $M_{\tilde{\chi}_{1}^{0}} / M_{A_{H}}=200 \mathrm{GeV}$ (dashed line), and $M_{T} / M_{\tilde{t}}=400 \mathrm{GeV}$ and $M_{\tilde{\chi}_{1}^{0}} / M_{A_{H}}=$ $66.76 \mathrm{GeV}$ (dotted line). (b) $A_{b}$ in LHT in the plane of two independent parameter, $f$ and $\lambda_{2}$. The dot represent our study point.

where $\theta_{\mathrm{tb}}$ is the angle between spin polarization of top and bottom quark in the rest frame of top quark, and the coefficient of $A_{b}$ is related to the spin vector $S^{\mu}$ via

$$
A_{b}=-\frac{m_{t}^{2}-2 m_{W}^{2}}{m_{t}^{2}+2 m_{W}^{2}} \hat{S}_{t}
$$

with

$$
\begin{aligned}
\hat{S}_{t}= & \frac{2 m_{t}\left(M_{T}^{2}-m_{t}^{2}+2 M_{A_{H}}^{2}\right)}{M_{A_{H}^{2}} N}\left|\vec{P}_{A_{H}}\right|\left(\left|f_{L}\right|^{2}-\left|f_{R}\right|^{2}\right) \\
= & \frac{\left(\left|f_{L}\right|^{2}-\left|f_{R}\right|^{2}\right)}{M_{A_{H}^{2}} N}\left(M_{T}^{2}-m_{t}^{2}+2 M_{A_{H}}^{2}\right) \\
& \times \sqrt{\left(M_{T}^{2}-\left(m_{t}-M_{A_{H}}\right)^{2}\right)\left(M_{T}^{2}-\left(m_{t}+M_{A_{H}}\right)^{2}\right)} .
\end{aligned}
$$

Here $\vec{P}_{A_{H}}$ is the three momentum of the LTP in the rest frame of top quark and $S_{t}^{2}=$ $-S^{\mu} S_{\mu}$. In the same way as SUSY, $A_{b}$ depends on $f_{L} / f_{R}$. In figure 07, we show $A_{b}$ with several values of masses and compare with results in SUSY. For SUSY, $A_{b}$ 's are calculated with formulas given in [47. We notice that $A_{b}$ in LHT is as sensitive as $A_{b}$ in SUSY, which has strong dependence on the ratio $f_{L} / f_{R}$, although they have different spin structure in the vertex, $T \rightarrow t A_{H}$. The statistical uncertainties in the measurement of $A_{b}$ is even better in the LHT since the production cross section is much larger than one in SUSY for given masses (see 47] for detail.). 


\section{Summary and conclusions}

In this paper, we studied the phenomenology of top partners in the context of the MSSM and LHT. In the MSSM, a discrete symmetry, R-parity, is introduced and the lightest super-particle is automatically stable. Its characteristic missing energy signals has been regarded as a genuine feature of the theory. Exactly the same signature can happen in the LHT where a discrete symmetry, dubbed T-parity, can play similar roles with those of the R-parity in the MSSM. Since the collider signals of both theories can mimic each other's, one need to compare their phenomenology precisely to understand the underlying physics.

We first estimated the total cross section of the pair production of top partners (the lightest scalar top quark in the MSSM and the T-odd top quark partner in the LHT) in the near future linear colliders where we can have better resolution for various observable quantities than that of the LHC and better chance to discriminate the "fake" theories. The larger cross section is expected for T-odd quark production than scalar top quark production once their masses are set to be exactly the same since T-odd quark has 2 times larger number of helicity degrees of freedom than that of scalar top quark (see figure 4). Also the angular distributions for T-odd quark (or its leptonic decay products) can be distinguishable from that of scalar top quark, again thanks to its fermionic characteristics (see figure 5). The standard model background was also estimated and we found that it is quite plausible for us to be able to distinguish the signals of LHT and MSSM from the ones of the standard model. We provide the analytic expressions for reconstruction of Top-partner's mass in the LHT and MSSM in the hadronic or semi-leptonic channel. The energy distribution of top quark (see figure 6) can be a useful observable quantity for study of top partners. The angular distribution of b-jet in the final state was studied since it can provide an important understanding about the helicity of the top-partner (see figure 7). In the appendix, we presented asymmetries for the LHT ( $A_{L R}$ and $\left.A_{F B}\right)$. They are found to be useful to understand the 'vectorlikeness' of the T-odd quark in the LHT using asymmetries.

In conclusion, linear collider experiments with the better resolution than that of hadronic collider experiments can provide a nice chance to probe and discriminate the competing theories beyond the standard model such as the LHT and MSSM which share similar phenomenological features.

\section{A. Left-right polarization asymmetry: $A_{L R}$}

With polarized electron beams the cross sections $\sigma_{L}$ and $\sigma_{R}$ for the scattering of lefthanded and right-handed electrons on unpolarized positrons can be separately measured. 

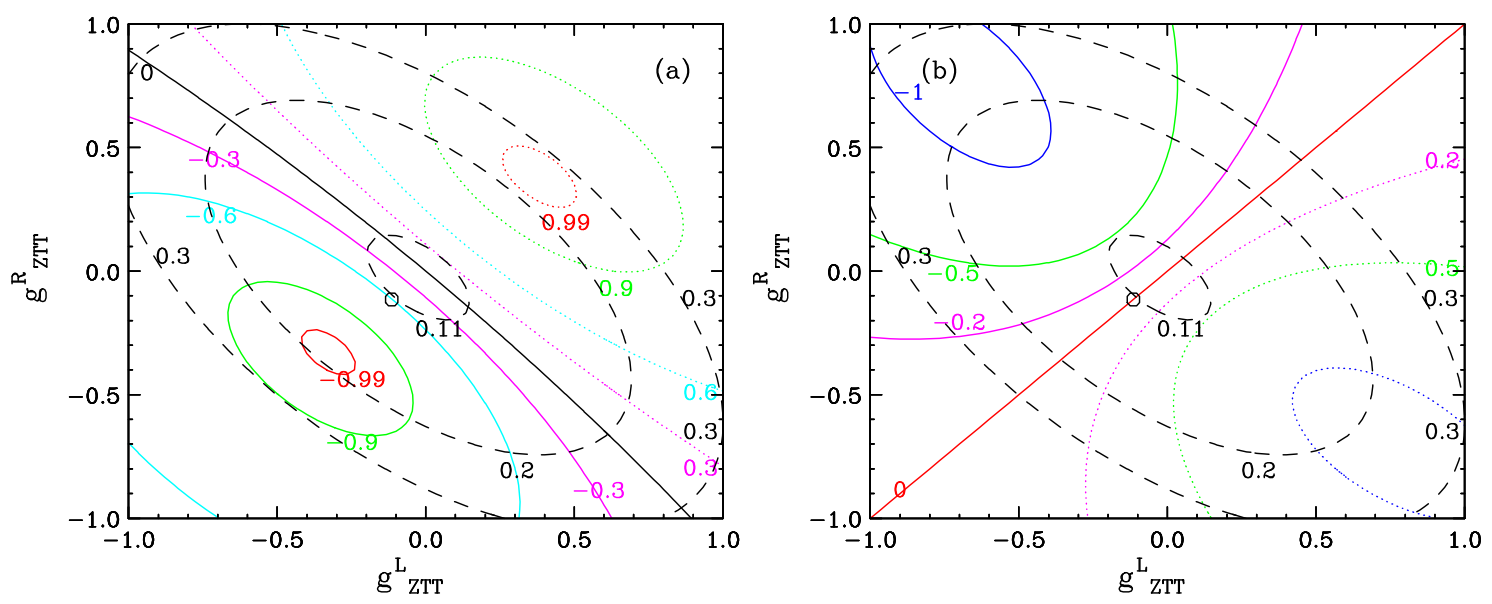

Figure 8: (a) The left-right asymmetry for polarized scattering and (b) the forward-backward asymmetry. Here we think of more generic case where the vector-like condition or $\left(G_{L L}=G_{L R}\right.$ and $\left.G_{R R}=G_{R L}\right)$ does not apply. For LHT, we get $A_{L R}=-0.6$ and $A_{F B}=0$.

The left-right polarization asymmetry $A_{L R}$ is defined as

$$
\begin{aligned}
A_{L R}^{L H T} & =\frac{\sigma_{L}-\sigma_{R}}{\sigma_{L}+\sigma_{R}} \\
& =\frac{\left(\left|G_{L L}\right|^{2}+\left|G_{L R}\right|^{2}-\left|G_{R R}\right|^{2}-\left|G_{R L}\right|^{2}\right)\left(1+\frac{1}{3} \beta^{2}\right)+2 R e\left(G_{L L} G_{L R}^{*}-G_{R R} G_{R L}^{*}\right)\left(1-\beta^{2}\right)}{\left(\left|G_{L L}\right|^{2}+\left|G_{L R}\right|^{2}+\left|G_{R R}\right|^{2}+\left|G_{R L}\right|^{2}\right)\left(1+\frac{1}{3} \beta^{2}\right)+2 R e\left(G_{L L} G_{L R}^{*}+G_{R R} G_{R L}^{*}\right)\left(1-\beta^{2}\right)} \\
& =\frac{(\mathcal{A}-\mathcal{B})\left(1+\frac{1}{3} \beta^{2}\right)+\mathcal{D}\left(1-\beta^{2}\right)}{(\mathcal{A}+\mathcal{B})\left(1+\frac{1}{3} \beta^{2}\right)+\mathcal{C}\left(1-\beta^{2}\right)},
\end{aligned}
$$

where $\mathcal{D}=2 \operatorname{Re}\left(G_{L L} G_{L R}^{*}-G_{R R} G_{R L}^{*}\right)$. Figure (a) shows contour lines for several values of $A_{L R}$ and the dot represent the prediction of LHT, $g_{Z T \bar{T}}^{L}=g_{Z T \bar{T}}^{R}=-\frac{2}{3} \frac{g}{c_{W}} s_{W}^{2}$. In this plot, we assume that the photon coupling is purely vector-like but we do not assume $G_{L L}=G_{L R}$ and $G_{R R}=G_{R L}$. Instead we take the coupling $\bar{T}_{-} \gamma_{\mu}\left(g_{Z T \bar{T}}^{L} P_{L}+g_{Z T \bar{T}}^{R} P_{R}\right) Z^{\mu} T_{-}$and vary $g_{Z T \bar{T}}^{L}$ and $g_{Z T \bar{T}}^{R}$. Solid lines represent negative asymmetries: $A_{L R}=-0.99,-0.9,-0.6$ and -0.3 while black solid line shows zero asymmetry and dotted lines represent positive asymmetries: $A_{L R}=0.99,0.9,0.6$ and 0.3. The total cross section is independent measurement and can be used to identify the couplings with the aid of this asymmetry. Dashed contour lines represent cross sections in pb.

\section{B. Forward-backward Asymmetry: $A_{F B}$}

The forward-backward asymmetry for unpolarized beams is defined to be the number of $T$ at CM scattering angle $\theta$ minus the number of $T$ at angle $\pi-\theta$ divided by the sum,

$$
A_{F B}(\theta)=\frac{d \sigma(\theta)-d \sigma(\pi-\theta)}{d \sigma(\theta)+d \sigma(\pi-\theta)}
$$


The integrated asymmetry is

$$
\begin{aligned}
A_{F B}^{L H T} & =\frac{\int_{0}^{\pi / 2}[d \sigma(\theta)-d \sigma(\pi-\theta)]}{\sigma} \\
& =\frac{N(\text { forward })-N(\text { backward })}{N(\text { forward })+N(\text { backward })} \\
& =\frac{\left(\left|G_{L L}\right|^{2}+\left|G_{R R}\right|^{2}-\left|G_{L R}\right|^{2}-\left|G_{R L}\right|^{2}\right) \beta}{\left(\left|G_{L L}\right|^{2}+\left|G_{L R}\right|^{2}+\left|G_{R R}\right|^{2}+\left|G_{R L}\right|^{2}\right)\left(1+\frac{1}{3} \beta^{2}\right)+2 R e\left(G_{L L} G_{L R}^{*}+G_{R R} G_{R L}^{*}\right)\left(1-\beta^{2}\right)} \\
& =\frac{\mathcal{A}-\mathcal{B}}{(\mathcal{A}+\mathcal{B})\left(1+\frac{1}{3} \beta^{2}\right)+\mathcal{C}\left(1-\beta^{2}\right)} .
\end{aligned}
$$

$A_{F B}=0$ (see figure $\left.8(\mathrm{~b})\right)$ since $\gamma$ and $Z$ couplings to $T \bar{T}$ are purely vector-like and hence $\mathcal{A}=\mathcal{B}$.

\section{C. $T_{+}$production at the ILC}

In this appendix, we consider possible production and detection of even parity top partner for the completeness. First, even parity top partner $\left(T_{+}\right)$is always heavier than odd parity one $\left(T_{-}\right)$.

$$
\frac{M_{T_{-}}}{M_{T_{+}}}=\frac{\lambda_{2}}{\sqrt{\lambda_{1}^{2}+\lambda_{2}^{2}}} \leq 1
$$

Contrast to the case at the LHC, where $T_{+}$could be singly produced via $t$-channel $\mathrm{W}$ boson exchange $\left(q b \rightarrow q^{\prime} T_{+}\right)$14, 15, $T_{+}$can only be produced by pair via $s$-channel photon and $\mathrm{Z}$ boson exchanges at the ILC. Here are the relevant Feynman rules describing $\gamma \bar{T}_{+} T_{+}$and $Z \bar{T}_{+} T_{+}$couplings.

$$
\mathcal{L}=\bar{T}_{+}\left[\frac{2}{3} \gamma_{\mu}\left(e A^{\mu}+\left(-\frac{g}{c_{w}} s_{w}^{2}+\frac{3}{4} \frac{g}{c_{w}} \frac{v^{2}}{f^{2}} c_{\lambda}^{4} P_{L}\right) Z^{\mu}\right)\right] T_{+},
$$

where $c_{\lambda}=\lambda_{1} / \sqrt{\lambda_{1}^{2}+\lambda_{2}^{2}}$ was introduced earlier. $T_{+}$mostly decays to $W b$ but it can also decay to $t H$ and $T_{-} A_{H}$ [12].

The cross section of $\bar{T}_{+} T_{+}$production is kinematically suppressed in comparison with that of $\bar{T}_{-} T_{-}$

$$
\frac{\sigma_{e^{+} e-\rightarrow \bar{T}_{+} T_{+}}}{\sigma_{e^{+} e^{-} \rightarrow \bar{T}_{-} T_{-}}} \simeq \frac{\sqrt{1-4 M_{T_{+}}^{2} / s}\left(1+2 M_{T_{+}} / s\right)}{\sqrt{1-4 M_{T_{-}}^{2} / s}\left(1+2 M_{T_{-}} / s\right)}
$$

with additional $\frac{v^{2}}{f^{2}} c_{\lambda}^{4}$ order corrections. For the linear collider with $\sqrt{s}=1 \mathrm{TeV}$, it is challenging to see the signals of T-even top quarks since they only can be produced near the threshold. Only very restrictive parameter space is available for $M_{T_{+}} \leq 500 \mathrm{GeV}$. 


\section{Acknowledgments}

We thank A. Pukhov and F. Maltoni for useful correspondence regarding many questions about CalcHEP and MadGraph/MadEvent. We are also grateful to C.P. Yuan and R. Kitano for discussion about the spin vector method, and M. Perelstein for reading our manuscript and useful comments. Fermilab is operated by Fermi Research Alliance, LLC under Contract No. DE-AC02-07CH11359 with the United States Department of Energy. SP is supported by BK21 program of Korean government.

\section{References}

[1] N. Arkani-Hamed, A.G. Cohen and H. Georgi, Electroweak symmetry breaking from dimensional deconstruction, Phys. Lett. B 513 (2001) 232 hep-ph/0105239.

[2] N. Arkani-Hamed, A.G. Cohen, E. Katz and A.E. Nelson, The littlest Higgs, JHEP 07 (2002) 034 hep-ph/0206021.

[3] M. Schmaltz and D. Tucker-Smith, Little Higgs review, Ann. Rev. Nucl. Part. Sci. 55 (2005) 229 hep-ph/0502182.

[4] M. Perelstein, Little Higgs models and their phenomenology, Prog. Part. Nucl. Phys. 58 (2007) 247 hep-ph/0512128.

[5] C. Csáki, J. Hubisz, G.D. Kribs, P. Meade and J. Terning, Big corrections from a little Higgs, Phys. Rev. D 67 (2003) 115002 hep-ph/0211124.

[6] C. Csáki, J. Hubisz, G.D. Kribs, P. Meade and J. Terning, Variations of little Higgs models and their electroweak constraints, Phys. Rev. D 68 (2003) 035009 hep-ph/0303236.

[7] J.L. Hewett, F.J. Petriello and T.G. Rizzo, Constraining the littlest Higgs, JHEP 10 (2003) 062 hep-ph/0211218.

[8] H.-C. Cheng and I. Low, TeV symmetry and the little hierarchy problem, JHEP 09 (2003) 051 hep-ph/0308199.

[9] H.-C. Cheng and I. Low, Little hierarchy, little Higgses and a little symmetry, JHEP 08 (2004) 061 hep-ph/0405243.

[10] I. Low, T parity and the littlest Higgs, JHEP 10 (2004) 067 hep-ph/0409025.

[11] J. Hubisz, P. Meade, A. Noble and M. Perelstein, Electroweak precision constraints on the littlest Higgs model with T parity, JHEP 01 (2006) 135 hep-ph/0506042].

[12] J. Hubisz and P. Meade, Phenomenology of the littlest Higgs with T-parity, Phys. Rev. D 71 (2005) 035016 hep-ph/0411264.

[13] G. Burdman, M. Perelstein and A. Pierce, Collider tests of the little Higgs model, Phys. Rev. Lett. 90 (2003) 241802 hep-ph/0212228.

[14] T. Han, H.E. Logan, B. McElrath and L.-T. Wang, Phenomenology of the little Higgs model, Phys. Rev. D 67 (2003) 095004 hep-ph/030104d.

[15] M. Perelstein, M.E. Peskin and A. Pierce, Top quarks and electroweak symmetry breaking in little Higgs models, Phys. Rev. D 69 (2004) 075002 hep-ph/0310039.

[16] S.C. Park and J. Song, Phenomenology of the heavy bh in a littlest Higgs model, Phys. Rev. D 69 (2004) 115010. 
[17] A. Birkedal, A. Noble, M. Perelstein and A. Spray, Little Higgs dark matter, Phys. Rev. D 74 (2006) 035002 hep-ph/0603077.

[18] J. Hubisz, S.J. Lee and G. Paz, The flavor of a little Higgs with T-parity, JHEP 06 (2006) 041 hep-ph/0512169.

[19] M. Blanke et al., Particle antiparticle mixing, $\epsilon_{k}, \Delta(\gamma(q)), A_{S L}(q), A_{C P}\left(B_{d} \rightarrow \psi k_{s}\right)$, $A_{C P}\left(B_{s} \rightarrow \psi \phi\right)$ and $B \rightarrow X_{s, d} \gamma$ in the littlest Higgs model with T-parity, JHEP 12 (2006) 003 hep-ph/0605214.

[20] C.-S. Chen, K. Cheung and T.-C. Yuan, Novel collider signature for little Higgs dark matter models, Phys. Lett. B 644 (2007) 158 hep-ph/0605314.

[21] M. Blanke et al., Another look at the flavour structure of the littlest Higgs model with T-parity, Phys. Lett. B 646 (2007) 253 hep-ph/0609284.

[22] A. Freitas and D. Wyler, Phenomenology of mirror fermions in the littlest Higgs model with T-parity, JHEP 11 (2006) 061 hep-ph/0609103.

[23] A. Belyaev, C.-R. Chen, K. Tobe and C.P. Yuan, Phenomenology of littlest Higgs model with T-parity: including effects of T-odd fermions, Phys. Rev. D 74 (2006) 115020 hep-ph/0609179.

[24] M. Carena, J. Hubisz, M. Perelstein and P. Verdier, Collider signature of T-quarks, Phys. Rev. D 75 (2007) 091701 hep-ph/0610156.

[25] P. Meade and M. Reece, Top partners at the LHC: spin and mass measurement, Phys. Rev. D 74 (2006) 015010 hep-ph/0601124.

[26] S. Matsumoto, M.M. Nojiri and D. Nomura, Hunting for the top partner in the littlest Higgs model with T-parity at the LHC, Phys. Rev. D 75 (2007) 055006 hep-ph/0612249.

[27] D. Choudhury and D.K. Ghosh, LHC signals of T-odd heavy quarks in the littlest Higgs model, hep-ph/0612299.

[28] V.D. Barger and R.J.N. Phillips, Collider physics, avaible onling.

[29] A. Pukhov, CalcHEP 3.2: MSSM, structure functions, event generation, batchs and generation of matrix elements for other packages, hep-ph/0412191.

[30] T. Stelzer and W.F. Long, Automatic generation of tree level helicity amplitudes, Comput. Phys. Commun. 81 (1994) 357 hep-ph/9401258].

[31] F. Maltoni and T. Stelzer, MadEvent: automatic event generation with MadGraph, JHEP 02 (2003) 027 hep-ph/0208156.

[32] S. Jadach and B.F.L. Ward, YFS2: the second order Monte Carlo for fermion pair production at lep/slc with the initial state radiation of two hard and multiple soft photons, Comput. Phys. Commun. 56 (1990) 351.

[33] M. Skrzypek and S. Jadach, Exact and approximate solutions for the electron nonsinglet structure function in QED, Z. Physik C 49 (1991) 577.

[34] A.K. Datta, K. Kong and K.T. Matchev, The impact of beamstrahlung on precision measurements at CLIC, hep-ph/0508161.

[35] CLIC Physics Working Group collaboration, E. Accomando et al., Physics at the CLIC multi-TeV linear collider, hep-ph/0412251. 
[36] M. Battaglia, A. Datta, A. De Roeck, K. Kong and K.T. Matchev, Contrasting supersymmetry and universal extra dimensions at the CLIC multi-TeV $e^{+} e^{-}$collider, JHEA 07 (2005) 033 hep-ph/0502041.

[37] M. Battaglia, A.K. Datta, A. De Roeck, K. Kong and K.T. Matchev, Contrasting supersymmetry and universal extra dimensions at colliders, hep-ph/0507284.

[38] S.Y. Choi, K. Hagiwara, H.U. Martyn, K. Mawatari and P.M. Zerwas, Spin analysis of supersymmetric particles, hep-ph/0612301.

[39] G.A. Moortgat-Pick et al., The role of polarized positrons and electrons in revealing fundamental interactions at the linear collider, hep-ph/0507011.

[40] T. Sjostrand et al., High-energy-physics event generation with PYTHIA 6.1, Comput. Phys. Commun. 135 (2001) 238 hep-ph/0010017.

[41] G.L. Kane, G.A. Ladinsky and C.P. Yuan, Using the top quark for testing standard model polarization and CP predictions, Phys. Rev. D 45 (1992) 124.

[42] A. Datta, K. Kong and K.T. Matchev, Discrimination of supersymmetry and universal extra dimensions at hadron colliders, Phys. Rev. D 72 (2005) 096006 hep-ph/0509246.

[43] A.J. Barr, Using lepton charge asymmetry to investigate the spin of supersymmetric particles at the LHC, Phys. Lett. B 596 (2004) 205 hep-ph/0405052.

[44] L.-T. Wang and I. Yavin, Spin measurements in cascade decays at the LHC, JHEP 04 (2007) 032 hep-ph/0605296.

[45] J.M. Smillie and B.R. Webber, Distinguishing spins in supersymmetric and universal extra dimension models at the large hadron collider, JHEP 10 (2005) 069 hep-ph/0507170.

[46] A. Alves, O. Eboli and T. Plehn, It's a gluino, Phys. Rev. D 74 (2006) 095010 hep-ph/0605067.

[47] R. Kitano, T. Moroi and S.-F. Su, Top-squark study at a future $e^{+} e^{-}$linear collider, JHEA 12 (2002) 011 hep-ph/0208149. 